\title{
Application of Solvent Demulsification-Dispersive Liquid-Liquid Microextraction Based on Solidification of Floating Organic Drop Coupled with High Perfomence Liquid Chromatography in Determination of Sulfonylurea Herbicides in Water and Soil
}

\author{
Li Yan, Zhu Jing, Ren Lin, Li Yongxin and Zou Xiaoli* \\ Department of Sanitary Technology, West China School of Public Health, Sichuan University 17, \\ Wuhou District, 610041 Chengdu, Sichuan, China
}

\begin{abstract}
A method for simultaneous determination of nine sulfonylurea herbicides in water and soil samples with solvent demulsification-dispersive liquid-liquid microextraction based on solidification of floating organic drop coupled with high perfomence liquid chromatography was established. $120 \mu \mathrm{L}$ of 1-dodecanol as extractant and $400 \mu \mathrm{L}$ of methanol as dispersing reagent were injected into the sample solution adjusted to $\mathrm{pH} 2.0$ to extract the target compounds for high performance liquid chromatography (HPLC) analysis. The detection limits-were $0.24-0.81 \mu \mathrm{g} \mathrm{L}-1$ for water samples and $2.42-8.12 \mu \mathrm{g} \mathrm{kg}^{-1}$ for soil samples, and the quantification limits were 0.79-2.69 $\mu \mathrm{g} \mathrm{L}^{-1}$ for water samples and 8.06-27.04 $\mu \mathrm{g} \mathrm{kg}^{-1}$ for soil samples respectively. The average recoveries were 78.9-104.8\% with the relative standard derivations (RSDs) of 2.73-10.4\% for water samples, and 75.5-107\% with the RSDs of 5.66-12.4\% for soil samples. The method had the advantages of little consumption of organic solvent, low cost and high enrichment factors.
\end{abstract}

Keywords: SFO-DLLME; HPLC; sulfonylurea herbicide

\section{Introduction}

Sulfonylurea herbicides, as a large family of herbicides widely used in agriculture, are characterized by broadspectrum weed control at low dosage, good crop selectivity and low mammalian toxicity. Even though sulfonylurea herbicides are decomposed rapidly in water and soil due to their thermal instability and chemical instability, they still remain in circumstance at a trace level. ${ }^{1-2}$ Much attention has been attached to the phytotoxicity of sulfonylurea herbicides residues. In 2004, a guideline entitled "Guideline for safety application of sulfonylurea

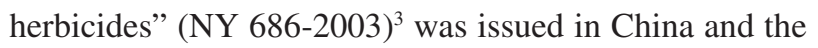
dosage, administration and sensitivity of crop varieties of 21 kinds of sulfonylurea herbicides were illustrated. maximum residue limits (MRLs) for theses herbicides have been established in many countries. Due to the fact that sulfonylurea herbicides residues in environment might be harmful to some animals and plants, it is necessary to develop a reliable analytical method for the detection of sulfonylurea herbicides residues in environment. Several analytical methods have been developed to determine sulfonylurea herbicide residues, including

*e-mail: zouxl_1113@163.com high performance liquid chromatography with ultraviolet detection (HPLC-UV), ${ }^{4-6}$ gas chromatography (GC), ${ }^{7}$ high performance liquid chromatography coupled to mass spectrometry (HPLC-MS), ${ }^{8}$ gas chromatography coupled to mass spectrometry (GC-MS) ${ }^{9}$ and so on. ${ }^{10}$ Since most of sulfonylurea herbicides were instable at high temperature, a time consuming procedure of sample derivatization or hydrolyzation was required prior to analysis with GC and GC-MS, which limited the application of GC and GC-MS in determination of sulfonylureas. HPLC-UV and HPLC-MS can be used for analysis despite the volatility or heat stability of the analytes, and can be used to directly analyze these herbicides without derivatization. HPLC-UV is a fast, simple, easy-to-use and widely available technique, which is usually chosen in the determination of sulfonylurea herbicides.

Since the amount of sulfonylurea herbicides in the environmental samples is at a trace level, the sample pretreatment is needed to extract and enrich them before analysis. Liquid-liquid extraction (LLE) and solidphase extraction (SPE) ${ }^{11}$ were the most commonly used techniques in sample pretreatments. LLE can offer high reproducibility and high sample capacity, but suffer from the disadvantages of time-consuming procedure and large volume of sample and toxic organic solvent. SPE 
can overcome some disadvantages of LLE, such as high consumption of both sample and organic solvent, but it is also time-consuming and relatively expensive. Some new pretreatment technology were used for analysis of sulfonylurea herbicides such as molecularly imprinted polymers extraction, ${ }^{12}$ nanostructured supramolecular solvent microextraction, ${ }^{13}$ magnetic solid-phase extraction ${ }^{14}$ and so on. ${ }^{15,16}$

Since 1990s, liquid-phase microextraction (LPME) has attracted increasing attention as a novel technique for sample pretreatment, and different modes of LPME have been developed in the past 20 years, such as single drop microextraction (SDME), hollow fiber based liquid-phase microextraction (HF-LPME), dispersive liquid-liquid microextraction (DLLME). ${ }^{17} \mathrm{~A}$ dispersive solvent is used in DLLME and a cloudy solution can be produced since extractant is dispersed in the samples with the help of dispersive solvent. Due to the large contact area between the extraction solvent and the sample, high extraction efficiency can be obtained within a short time. However, it is not easy to collect the extractant after extraction in DLLME since the density of halohydrocarbons as common extractant solvent is higher than water and will sink to the bottom of tube. In addition, most halohydrocarbons are highly toxic, environmentally unfriendly and cannot be directly injected into HPLC for analysis. Therefore, a new technique based on the solidification of a floating organic drop and LPME (SFO-LPME), was reported by Zanjani et al. ${ }^{18} \mathrm{~A}$ low density and less toxic solvent with proper melting point is used as the extraction solvent in this technique. Meanwhile, in order to obtained higher extraction efficiencies, Leong and Huang ${ }^{19}$ introduced another microextraction method combined the solidification of a floating organic drop with DLLME (SFO-DLLME) in 2008. Compared to DLLME, SFO-DLLME has the properties of simple operation, low cost, rapid speed, high efficiency, as well as lower toxic and environmentally friendly. 1-octanol and dihexyl ether were exploited as extraction solvents to extract the sulfonylurea herbicides in water and soil samples, also combined with HPLC-UV analysis. In the experiment, surfactant was used as emulsifier to enhance the dispersion of extraction solvent. ${ }^{20}$

SFO-DLLME has the advantages of DLLME of high extraction efficiency, but suffers the same disadvantage as DLLME that centrifugation will be needed to gather the dispersed extraction solvent and separate the organic and aqueous phases, which is time-consuming and restricts the application of DLLME in field processing and automated analysis. In 2010, Zacharis et al. ${ }^{4}$ proposed a method of solvent-based demulsification dispersive liquid-liquid microextraction (SD-DLLME). Instead of centrifugation, demulsification is taken to separate the organic and aqueous phases after the extraction. A certain amount of organic solvent was added as demulsifier to change the interfacial adhesion between the organic and aqueous phases, surface tension and Van der Waal's forces, then the emulsion system will be broken and extraction solvent droplets will be gathered. This method improves the mode of phase separation of SFO-DLLME to make it possible to be used in field processing and automated analysis without electric centrifugal equipment. ${ }^{21}$ It has been successfully applied in the determination of organic and inorganic compounds such as phosphate, ${ }^{22}$ heavy metals, ${ }^{23}$ amphetamines, ${ }^{24}$ organochlorine pesticides, ${ }^{25}$ polycyclic aromatic hydrocarbons. ${ }^{26}$

In this paper, the method of SD-SFO-DLLME (solventbased demulsification and solidification of a floating organic drop combined with dispersive liquid-liquid microextraction) with HPLC-UV analysis was developed for the determination of sulfonylurea herbicide residues in water and soil samples.

\section{Experimental}

\section{Instrumentation}

AKTA Purifier 900 high performance liquid chromatography equipped with an ultraviolet detector (GE Healthcare Bio-Sciences, Uppsala, Sweden) was used to identify and quantify the target compounds in this study. The separation was performed on a Venusil $\mathrm{MP} \mathrm{C}_{18}$ column (250 mm $\times 4.6 \mathrm{~mm}, 5 \mu \mathrm{m}$, Agela Technologies, Wilmington, USA) at $30{ }^{\circ} \mathrm{C}$ with the flow rate of $1.0 \mathrm{~mL} \mathrm{~min}^{-1}$ and the detection was carried out at the wavelength of $240 \mathrm{~nm}$. The gradient elution mode was employed. Phase A consisted of acetonitrile and methanol (50:50, v/v) and phase B was $0.001 \mathrm{~mol} \mathrm{~L}^{-1}$ aqueous hydrochloric acid solution $(\mathrm{pH}=3.0)$. The elution procedure was as follows: $55 \%$ A for 0-4.15 min, linear gradient from 55\% to $90 \%$ A for 4.15-16.60 $\mathrm{min}, 90 \%$ A for $16.60-20.75 \mathrm{~min}, 55 \% \mathrm{~A}$ for 20.75-24.90 $\mathrm{min}$.

\section{Reagents}

Nicosulfuron (95.5\%), thifensulfuron-methyl (97.0\%), metsulfuron-methyl (99.5\%), mesosulfuron-methyl (99.0\%), tribenuron-methyl $(99.0 \%)$, bensulfuron-methyl $(98.5 \%)$ and pyrazosulfuron-methyl $(97.5 \%)$ were purchased from Dr. Ehrenstorfer GmbH (Augsburg, Germany). procarbazone sodium $(99.0 \%)$ and chlorsulfuron $(99.0 \%)$ were obtained from Sigma Aldrich (St. Louis, MO, USA).

Sulfonylurea herbicides were dissolved in methanol with $0.1 \%$ of ammonia by ultrasonic assisted dissolution to make 
the stock solutions at a concentration of $1.0 \mathrm{mg} \mathrm{mL}^{-1}$. The stock solutions were sealed and stored at $-20^{\circ} \mathrm{C}$. Acetonitrile, methanol, dodecanol, hexadecane and undecanol were HPLC grade. Other reagents were analytical reagent grade unless otherwise noted. Ultrapure water $(18.2 \mathrm{M} \Omega \mathrm{cm})$ prepared by a Synergy 185 ultrapure water generator (Millipore, Bedford, USA) was used throughout.

Water samples were adjusted to $\mathrm{pH} 8.0$ with ammonia and stored at $4{ }^{\circ} \mathrm{C}$. Soil samples, according to The Technical Specification for soil Environmental monitoring of China (HJ166-2004), ${ }^{27}$ were stored in the glass bottle at $4{ }^{\circ} \mathrm{C}$.

\section{Extraction procedure}

\section{Water samples}

$20 \mathrm{~mL}$ of water sample adjusted to $\mathrm{pH} 2.0$ using $1 \mathrm{~mol} \mathrm{~L}^{-1}$ of $\mathrm{HCl}$ was placed in a capped glass centrifuge tube with conical bottom. $7 \mathrm{~g}$ of $\mathrm{NaCl}$ were added and dissolved to obtain a proper ionic strength. $120 \mu \mathrm{L}$ of undecanol as the extraction solvent and $400 \mu \mathrm{L}$ of methanol as the dispersive solvent were pipetted into the solution. The cloudy solution was formed and water bathed at $30{ }^{\circ} \mathrm{C}$ for $1 \mathrm{~min}$. After extraction, $1200 \mu \mathrm{L}$ of acetone were injected into the mixture as the demulsifier in order to make the organic solvent float on the surface of sample solution and was incubated in an ice bath so that the extractant was solidified in $5 \mathrm{~min}$. The solidified organic solvent was transferred to an EP tube with a small spoon and was melted at room temperature. After being diluted to $200 \mu \mathrm{L}$ with methanol and centrifuged at $15000 \mathrm{rpm}$ for $5 \mathrm{~min}$ to eliminate particles, $10 \mu \mathrm{L}$ of the supernatant (a mixture of extraction solvent undecanol and methanol) were injected into HPLC-UV system.

\section{Soil samples}

According to the guideline entitled "Soil Testing Part 1: Soil Sampling, processing and reposition" (NY/T 1121.1-2006), ${ }^{28} 5 \mathrm{~g}$ of dry soil samples passed through a 60 mesh sieve were mixed with $1.0 \mathrm{~mL}$ of methanol, and diluted to $50 \mathrm{~mL}$ with $0.1 \mathrm{~mol} \mathrm{~L}^{-1}$ of $\mathrm{NaHCO}_{3}$. After being ultrasonicated for $30 \mathrm{~min}$ and centrifuged at $3000 \mathrm{rpm}$ for $10 \mathrm{~min}, 20 \mathrm{~mL}$ of the supernatant was used for the following extraction procedures as described for water samples. At the same time, the water content of sample was determined.

\section{Calibration curves}

\section{Water sample}

The ultrapure water samples were spiked with different concentrations of sulfonylurea herbicides from $0.010 \mathrm{mg} \mathrm{L}^{-1}$ to $1.00 \mathrm{mg} \mathrm{L}^{-1}$, and processed by the procedures as described for water samples, SFO-DLLME and HPLC-UV analysis. To establish the calibration curve, the peak areas of sulfonylurea herbicides were plotted against the concentration of analytes.

\section{Soil sample}

Seven blank dry soil samples of $5 \mathrm{~g}$ were sonicated and centrifugated by the procedure as described for soil samples to obtain blank sample solutions. Then, the blank sample solutions were spiked and analyzed following the procedures described before.

\section{Spiked sample}

Three water samples with different contents were spiked with the standard solutions at $0.01,0.02,0.05 \mu \mathrm{g} \mathrm{mL}^{-1}$. For each concentration, 6 parallel spiked simulated samples were extracted at the same time following the procedures described before. 6 paralled spiked soil samples $(0.05$, $0.10,0.20 \mathrm{mg} \mathrm{kg}^{-1}$ ) were extracted following procedure described for soil samples.

\section{Optimization of extraction conditions}

In the optimization experiments, $20 \mathrm{~mL}$ of simulated water samples added with sulfonylurea herbicides at a concentration of $0.5 \mu \mathrm{g} \mathrm{mL} \mathrm{m}^{-1}$ were used throughout, and analyzed by HPLC-UV. Single factor experimental design was used in the optimization of extraction conditions.

\section{Results and Discussion}

\section{Optimization of chromatographic conditions}

The mixtures of organic solvent and acid solution (hydrochloric acid-formic acid, acetic acid, phosphoric acid) usually serve as mobile phase in HPLC-UV analysis of sulfonylurea herbicides since their $\mathrm{pKa}$ values were in range of 3.3-5.2. In this study, methanol and acetonitrile were investigated as organic phase at first. Aqueous hydrochloric acid solution $\left(0.001 \mathrm{~mol} \mathrm{~L}^{-1}\right)$ was used as phase B throughout the experiment. There was a difficulty in distinguishing chlorsulfuron with tribenuron-methyl when methanol was employed. In order to obtain the effective separation, the proportion of methanol had to be adjusted to less than $50 \%$, thereby prolonging the analysis time to more than $40 \mathrm{~min}$. The better elution effect was offered with acetonitrile, but chlorsulfuron and mesosulfuron-methyl, tribenuron-methyl and bensulfuron-methyl could not be effectively separated. Therefore, the mixture of acetonitrile and methanol (50:50, v/v) was chosen as the organic elution solution (phase A). 
Dissociatition of sulfonylurea herbicides will be influenced by acidity of mobile phase, which leads to show different chromatographic behaviors. An acidic mobile phase can be used to reduce dissociatition of analytes molecules to obtain better peaks. Therefore, several concentrations of $\mathrm{HCl}$ in the range of $0-0.05 \mathrm{~mol} \mathrm{~L}^{-1}$ were investigated while a proportion of 50:50 (v/v) of acetonitrile and methanol was used as organic phase. Tailing of the peaks occured without hydrochloric acid solution. As the higher concentration of hydrochloric acid solution was used, the peak profile became better. The symmetrical peaks of sulfonylurea herbicides were obtained until the concentration of hydrochloric acid solution was increased to more than $0.001 \mathrm{~mol} \mathrm{~L}^{-1}$. Considering the fact that the suitable $\mathrm{pH}$ values for $\mathrm{C}_{18}$ column were in the range of 1.5-8.5, $0.001 \mathrm{~mol} \mathrm{~L}^{-1} \mathrm{HCl}$ was used in further experiment.

In the study, the analytes were separated by isocratic elution initially. When the ratio of phase A to phase B was between $60: 40$ and 55:45 (v/v), all the nine analytes could be separated, but the retention time of procarbazone sodium and pyrazosulfuron-methyl was delayed to more than $40 \mathrm{~min}$. In order to obtain good separation within a short analytical time, the gradient elution procedure was considered 55\% A for 0-4.15 min, linear gradient from 55\% to $90 \%$ A for $4.15-16.60 \mathrm{~min}, 90 \%$ A for $16.60-20.75 \mathrm{~min}$, $55 \%$ A for 20.75-24.90 min. Under the conditions, the satisfactory separation for all the analytes was achieved within 25 min.

The wavelengths from $210-260 \mathrm{~nm}$ were investigated. It was found that the detection sensitivity decreased with the increase of wavelength, so did the baseline noise in HPLC-UV analysis. When the detection wavelength was $240 \mathrm{~nm}$, the detection sensitivity could meet the requirement of herbicides analysis and a stable baseline was obtained. The chromatogram obtained under the optimum condition was shown in Figure 1.

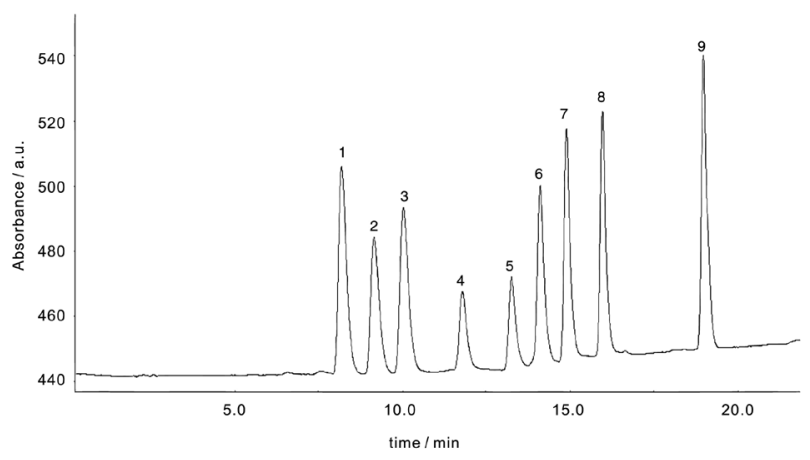

Figure 1. Chromatogram of standard solution for sulfonylurea herbicides. (1) nicosulfuron; (2) thifensulfuron-methyl; (3) metsulfuro-methyl; (4) chlorsulfuron; (5) mesosulfuron-methyl; (6) tribenuron-methyl; (7) bensulfuron-methyl; (8) procarbazone sodium; (9) pyrazosulfuronethyl.

\section{Optimization of the extraction conditions}

\section{Effect of the extraction solvent}

The most commonly used extraction solvent in SFO-DLLME, 1-dodecanol, 1-undecanol and hexadecane were studied in this paper. The extraction procedure was similar to that for water samples. The results showed that the extraction efficiency was lower than $20 \%$ with hexadecane due to its low polarity, and the analytes could be effectively extracted by 1-dodecanol and 1-undecanol except nicosulfuron and thifensulfuron-methyl (extraction efficiencies of nocosulfuron and thifensulfuron were 69.5-74.7\% and $67.3-71.5 \%$ for 1-dodecanol, $78.4-81.8 \%$ and $75.8-79.2 \%$ for 1-undecanol). 1-undecanol was selected as the extractant because nicosulfuron and thifensulfuronmethyl could obtain better extraction efficiencies.

To study the effect of the volume of the extraction solvent on the determination of the sulfonylurea herbicides, different amounts of 1-undecanol in the range of 30$150 \mu \mathrm{L}$ were investigated. The extraction efficiency was improved with the increase of the volume of 1-undecanol and it became constant until the volume of 1-undecanol was $120 \mu \mathrm{L}$. As a result, $120 \mu \mathrm{L}$ of extraction solvent was selected considering the extraction efficiencies and the enrichment factor.

\section{Effect of the dispersive solvent}

The dispersive solvent should be soluble both in water phase and organic phase so that the extraction solvent could be mixed with the sample solution and a homogeneous emulsion could be formed. In this study, methanol, acetonitrile and acetone were investigated and the extraction efficiencies were determined. It was demonstrated that methanol and acetone were better than acetonitrile except for tribenuron-methyl. Methanol performed better than acetone for tribenuron-methyl: the extraction efficiency was $75 \%$ for acetone, $80 \%$ for methanol and $85 \%$ for acetonitrile, respectively. Consequently, methanol was selected as the dispersive solvent.

Various volumes of methanol in the range of $50-1000 \mu \mathrm{L}$ were studied to find a suitable dosage. The results showed that the extraction efficiencies of sulfonylurea herbicides was enhanced as the methanol volume increased generally. When the volume of methanol was in the range of $300-450 \mu \mathrm{L}$, the extraction efficiencies were kept stable. However, when the volume was higher than $500 \mu \mathrm{L}$, the extraction efficiencies were slightly decreased, since the solubility of analytes in water might be increased with increasing amount of dispersive solvent. Therefore, $400 \mu \mathrm{L}$ of extraction solvent was used in the experiments considering the extraction efficiency and the emulsification effect. 


\section{Effect of $\mathrm{pH}$ of the sample}

Sulfonylurea herbicides are weak acids which $\mathrm{pK}_{\mathrm{a}}$ is 3.3-5.2. They are soluble in basic solutions. According to the references, ${ }^{8,9} 0.1$ mol L-1 of $\mathrm{NaHCO}_{3}$ containing $2 \%$ methanol was selected as the extraction solvent of soil samples in the experiment and the recoveries of $75-110 \%$ were obtained.

The experiments were carried out for the simulated water sample whose $\mathrm{pH}$ value was adjusted from 1.0 to 7.0 by $1 \mathrm{~mol} \mathrm{~L}^{-1}$ of $\mathrm{HCl}$. As shown in Figure.2, the extraction efficiency remained stable when $\mathrm{pH}$ was in the range of 1.0-3.0, then there was a dramatic decrease when $\mathrm{pH}$ value of the sample was more than 3.0. Thus, the extraction procedure was carried out under the acid medium of $\mathrm{pH} 2.0$.

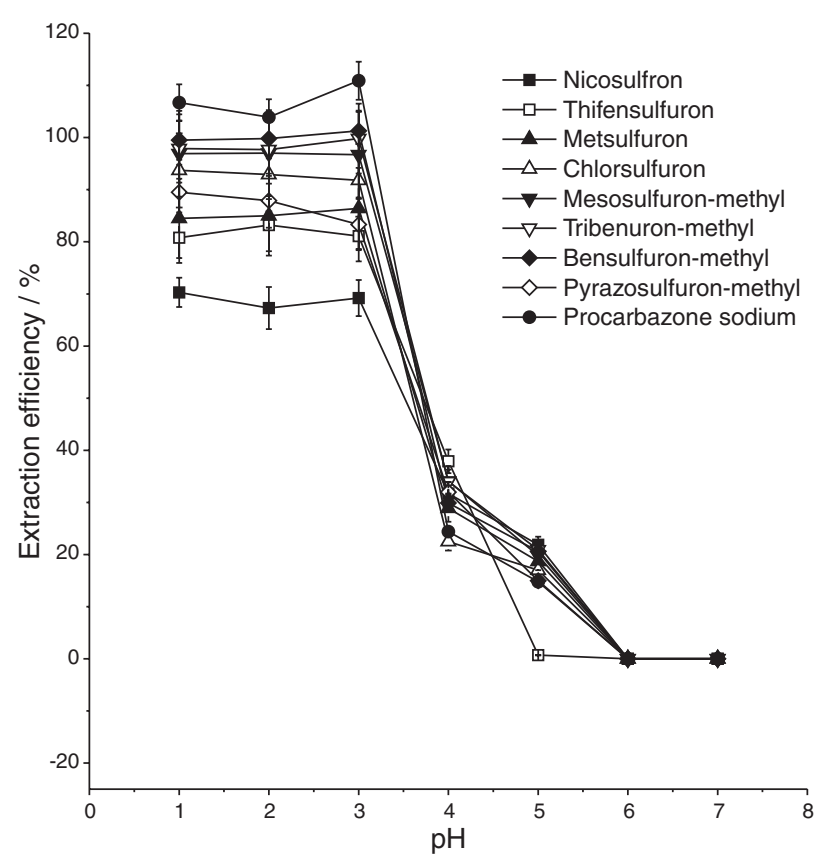

Figure 2. The effects of $\mathrm{pH}$ of extraction on the extraction efficiencies $(n=3)$.

\section{Effect of sodium chloride}

The addition of sodium chloride can affect the extraction efficiency by changing the ionic strength of the aqueous phase. In this work, the effect of sodium chloride on the extraction efficiencies of the analytes was assessed by adding different amounts of sodium chloride into the simulation samples, with the concentration of sodium chloride in the range of $0-0.35 \mathrm{~g} \mathrm{~mL}^{-1}$. The extraction efficiencies were enhanced as the concentration of sodium chloride increased and reached their peak values at $0.30 \mathrm{~g} \mathrm{~mL}^{-1}$ except that of nicosulfuron, which increased to $80 \%$ when the concentration of sodium chloride was $0.35 \mathrm{~g} \mathrm{~mL}^{-1}$. As a result, the saturated concentration of sodium chloride in sample solution was chosen as the optimal experimental condition.

\section{Effect of extraction temperature and time}

To study the influence of extraction temperature on the performance of extraction, various temperatures in the range of $25-70{ }^{\circ} \mathrm{C}$ were investigated. It was found that extraction efficiencies did not show significant changes when the temperature was below $35^{\circ} \mathrm{C}$, but decreased with further increase of temperature, which was attributed to the thermal instability of sulfonylurea herbicides. Considering degradation of sulfonylureas herbicides and the melting point of 1-undecanol, $30^{\circ} \mathrm{C}$ was selected as the extraction temperature.

The extraction time is defined between the beginning of water bath and the injection of acetone. The extraction time from 0-5 min were investigated. As a result, the extraction efficiency reached the peak value in water bath at $30{ }^{\circ} \mathrm{C}$ for $1 \mathrm{~min}$ and tended to remain stable. The analytes were allowed to be rapidly transfered from water sample to organic phase due to addition of dispersive solvent.

\section{Effect of demulsifier}

Demulsifier can be used to breakdown the emulsion system and accelerate phase separation by changing the interfacial adhesion between the organic and aqueous phases. It was found that good phase separation could be obtained while methanol, acetonitrile, and acetone were used as emulsifier, and recovery rate of acetone was better. Therefore, acetone was chosen as emulsifier. Then effect of volume of acetone was studied in range of 500-1500 $\mu \mathrm{L}$. The extraction efficiencies enhanced as the volume of acetone increasing. When the volume of acetone was $1200 \mu \mathrm{L}$, the extraction efficiencies of 9 kinds of sulfonylurea herbicides reached the maximum and good phase seperation were also found. Further increase of the volume of acetone led to slight decrease of extraction efficiencies. So $1200 \mu \mathrm{L}$ of acetone were used as demulsifier to separate the organic and aqueous phases. Extraction efficiency of sulfonylurea herbicides of demulsification was compared to that of centrifugation, and the relative deviations (RDs) were in range of $4.27-8.54 \%$.

\section{Method validation}

The calibration curves were constructed according Experimental section. As shown in Table 1, good linearity was observed in the range of $0.005-1.0 \mathrm{mg} \mathrm{L}^{-1}$. The method detection limits (MDLs) were calculated by the Hubaux-Vos (H-V) method based on the calibration curve mentioned above. Compared with the three fold signal-to-noise ratio ( $3 \mathrm{~S} / \mathrm{N}$ ) method, $\mathrm{H}-\mathrm{V}$ method is more reasonable to estimate MDLs since it can not only evaluate the uncertainty of the instrumental analysis but also consider the deviation brought by the sample pretreatment 
procedure. For the analysis of water sample, MDLs were in range of 0.24-0.81 $\mu \mathrm{g} \mathrm{L}-1$ and the method quantitative limits (MQLs) were in range of $0.81-2.70 \mu \mathrm{g} \mathrm{L}^{-1}$ when the amount of samples was $20 \mathrm{~mL}$. For the analysis of soil sample, MDLs were in range of 2.42-8.12 $\mu \mathrm{g} \mathrm{kg}^{-1}$ and MQLs were in range of 8.06-27.04 $\mathrm{g} \mathrm{kg} \mathrm{k}^{-1}$ when the amount of samples was $5 \mathrm{~g}$.
A certain standard solution at the concentration of $5 \mu \mathrm{g} \mathrm{mL} \mathrm{m}^{-1}$ was detected 6 times within one day and daily for 6 days. As a result, intra-day and inter-day RSDs were $1.19-5.42 \%$ and $2.16-6.04 \%$, respectively.

The recoveries of spiked samples were determined to evaluate the matrix influence on the method. As shown in Table 2, the recoveries were from $78.9-105 \%$ and the

Table 1. The linear regression, MDLs and MQLs of the method

\begin{tabular}{|c|c|c|c|c|}
\hline \multirow{3}{*}{ Analyte } & \multirow{3}{*}{ Calibration curve } & \multirow{3}{*}{ Correlation coefficent } & $\mathrm{MDL}^{\mathrm{a}}$ & $\mathrm{MQL}^{\mathrm{b}}$ \\
\hline & & & Water & Soil \\
\hline & & & $\left(\mu \mathrm{g} \mathrm{L}^{-1}\right) ;\left(\mu \mathrm{g} \mathrm{kg}^{-1}\right)$ & $\left(\mu \mathrm{g} \mathrm{L}^{-1}\right) ;\left(\mu \mathrm{g} \mathrm{kg}^{-1}\right)$ \\
\hline Nicosulfuron & $y=63.05 x-0.0559$ & 0.9944 & $0.38 ; 3.82$ & $1.27 ; 12.72$ \\
\hline Thifensulfuron-methyl & $y=44.96 x+0.0704$ & 0.9984 & $0.46 ; 4.62$ & $1.53 ; 15.38$ \\
\hline Metsulfuro-methyl & $y=52.82 x-0.0538$ & 0.9950 & $0.42 ; 4.22$ & $1.40 ; 14.05$ \\
\hline Chlorsulfuron & $y=35.43 x+0.0270$ & 0.9963 & $0.80 ; 8.04$ & $2.66 ; 26.84$ \\
\hline Mesosulfuron-methyl & $y=35.07 x-0.0348$ & 0.9984 & $0.81 ; 8.12$ & $2.70 ; 27.04$ \\
\hline Tribenuron-methyl & $y=42.64 x+0.0874$ & 0.9981 & $0.49 ; 4.89$ & $1.63 ; 16.32$ \\
\hline Bensulfuron-methyl & $y=47.62 x+0.0366$ & 0.9993 & $0.40 ; 4.02$ & $1.33 ; 13.39$ \\
\hline Procarbazone sodium & $y=52.75 x-0.0691$ & 0.9994 & $0.38 ; 3.85$ & $1.27 ; 12.82$ \\
\hline Pyrazosulfuron-methyl & $\mathrm{y}=71.51 \mathrm{x}-0.0370$ & 0.9993 & $0.24 ; 2.42$ & $0.81 ; 8.06$ \\
\hline
\end{tabular}

${ }^{\mathrm{a}} \mathrm{MDLs}=$ method detection limits; ${ }^{\mathrm{b}} \mathrm{MQLs}=$ method quantitative limits.

Table 2. The spiked recoveries of the water samples $(n=6)$

\begin{tabular}{|c|c|c|c|c|c|}
\hline Analyte & Background / $\left(\mu \mathrm{g} \mathrm{mL}^{-1}\right)$ & Spiked / $\left(\mu \mathrm{g} \mathrm{mL} L^{-1}\right)$ & Mean Found / $\left(\mu \mathrm{g} \mathrm{mL}^{-1}\right)$ & Recoveries / \% & $\operatorname{RSDs}^{\mathrm{b}} / \%$ \\
\hline & & 0.01 & 0.008 & 78.9 & 10.4 \\
\hline \multirow[t]{3}{*}{ Nicosulfuron } & $\mathrm{ND}^{\mathrm{a}}$ & 0.02 & 0.018 & 88.0 & 5.57 \\
\hline & & 0.05 & 0.048 & 96.4 & 5.36 \\
\hline & & 0.01 & 0.008 & 82.0 & 6.84 \\
\hline \multirow[t]{3}{*}{ Thifensulfuron-methyl } & ND & 0.02 & 0.018 & 91.0 & 5.97 \\
\hline & & 0.05 & 0.049 & 97.2 & 5.37 \\
\hline & & 0.01 & 0.008 & 78.2 & 7.93 \\
\hline \multirow[t]{3}{*}{ Metsulfuro-methyl } & ND & 0.02 & 0.018 & 89.5 & 7.74 \\
\hline & & 0.05 & 0.046 & 91.0 & 5.28 \\
\hline & & 0.01 & 0.008 & 82.1 & 6.65 \\
\hline \multirow[t]{3}{*}{ Chlorsulfuron } & ND & 0.02 & 0.020 & 101 & 6.57 \\
\hline & & 0.05 & 0.050 & 99.2 & 5.03 \\
\hline & & 0.01 & 0.008 & 83.5 & 8.12 \\
\hline \multirow[t]{3}{*}{ Mesosulfuron-methyl } & ND & 0.02 & 0.016 & 81.0 & 6.37 \\
\hline & & 0.05 & 0.051 & 102 & 6.47 \\
\hline & & 0.01 & 0.008 & 83.0 & 5.73 \\
\hline \multirow[t]{3}{*}{ Tribenuron-methyl } & ND & 0.02 & 0.020 & 97.5 & 5.74 \\
\hline & & 0.05 & 0.052 & 105 & 4.84 \\
\hline & & 0.01 & 0.019 & 74.0 & 8.40 \\
\hline \multirow[t]{3}{*}{ Bensulfuron-methyl } & 0.012 & 0.02 & 0.031 & 92.5 & 7.17 \\
\hline & & 0.05 & 0.063 & 102 & 8.68 \\
\hline & & 0.01 & 0.008 & 82.2 & 5.84 \\
\hline \multirow[t]{3}{*}{ Procarbazone sodium } & ND & 0.02 & 0.016 & 79.0 & 6.97 \\
\hline & & 0.05 & 0.052 & 104 & 5.17 \\
\hline & & 0.01 & 0.009 & 92.4 & 3.39 \\
\hline \multirow[t]{2}{*}{ Pyrazosulfuron-methyl } & ND & 0.02 & 0.020 & 97.5 & 3.74 \\
\hline & & 0.05 & 0.050 & 100 & 2.73 \\
\hline
\end{tabular}

${ }^{\mathrm{a}} \mathrm{ND}=$ not detected $;{ }^{\mathrm{b}} \mathrm{RSDs}=$ relative standard derivations. 
RSDs were from $2.73-10.4 \%$. As well, the recoveries of soil samples were from $75.5-107 \%$ and the RSDs were from $5.66-12.4 \%$.

\section{Stability of samples}

The spiked simulation samples adjusted to $\mathrm{pH} 8$ with aqueous solution of ammonia were stored at $4{ }^{\circ} \mathrm{C}$ and divided into 4 groups, 6 samples for each group, to evaluate the sample stability. The samples were determined on the first, third, seventh and fourteenth day, respectively. The mean determination values for every group were compared to those of the first group. A relative deviation of $3.79-8.29 \%$ was obtained, indicating that no degradation of sulfonylurea herbicides was observed for at least 2 weeks under the preservation conditions in the experiment.

\section{Sample analysis}

Twelve river water samples, ten farmland water samples and fifteen tap water samples around Chengdu City in Sichuan province of China were collected and analyzed with the proposed method. According to IUPAC: Harmonized Guidelines for Internal Quality Control in Analytical Chemistry Laboratories, ${ }^{29}$ a blank sample and a spiked sample as quality control material were inserted at an frequency of 1 per 10 samples analyzed. As a result, peaks of analytes did not appear in chromagrams of blank samples, and recoveies of spiked samples were in the range of $\mu \pm 2 \sigma(\mu=$ mean recovery, $\sigma=$ standard deviation), which suggested the experimental results were reliable. Bensulfuron-methyl was detected in one of the farmland water samples and the content was $12.02 \mu \mathrm{g} \mathrm{L}^{-1}$. The chromatogram of the real water sample and spiked water sample were shown in Figure. 3.

Four soil samples from suburban areas of Chengdu City were analyzed and one of them was found to contain chlorsulfuron, bensulfuron-methyl and procarbazone sodium of $39.64,33.14$ and $20.09 \mu \mathrm{g} \mathrm{kg}^{-1}$, respectively. The chromatogram of the real soil sample and spiked soil sample were shown in Figure. 4.

\section{Conclusions}

The proposed method of SD-SFO-DLLME coupled with HPLC-UV detection for the determination of sulfonylurea herbicides residues was simple, unexpensive and sensitive. Compared with traditional extraction methods such as LLE and SPE, SFO-DLLME achieves simultaneous completion of purification and enrichment, which makes it more rapid and simple. Compared with traditional SFO-DLLME, phase separation was performed by introducing of demulsifier without centrifugation.
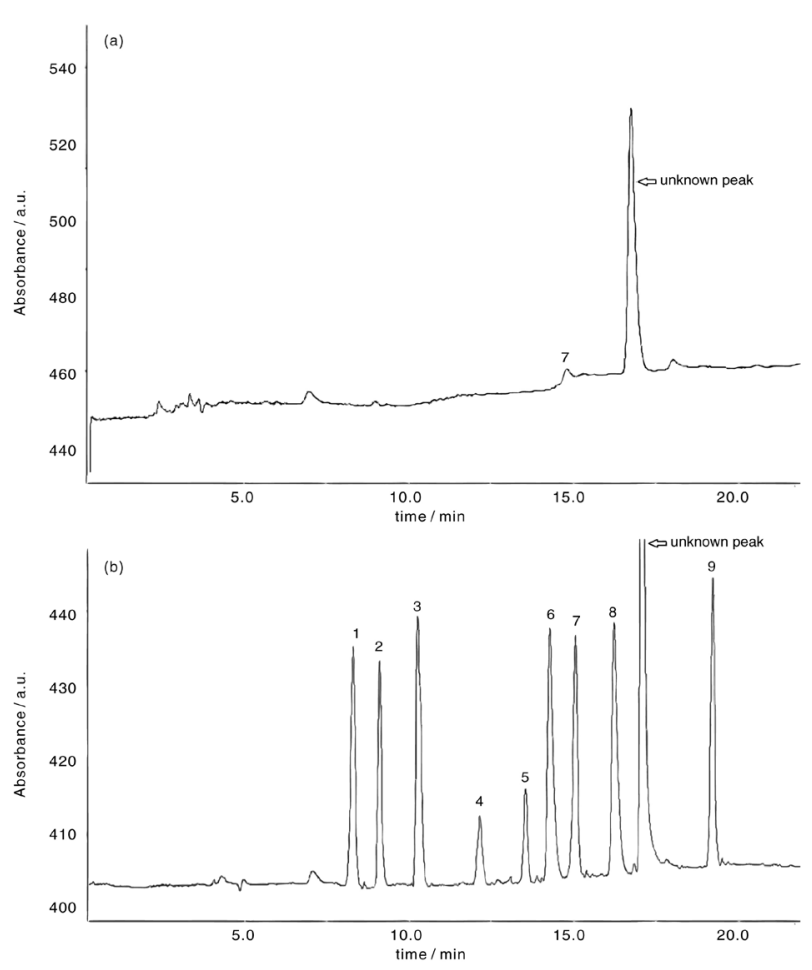

Figure 3. Chromatogram of (a) farmland water sample solution and (b) spiked water sample. (1) nicosulfuron; (2) thifensulfuron-methyl; (3) metsulfuro-methyl; (4) chlorsulfuron; (5) mesosulfuron-methyl; (6) tribenuron-methyl; (7) bensulfuron-methyl; (8) procarbazone sodium; (9) pyrazosulfuron-ethyl.
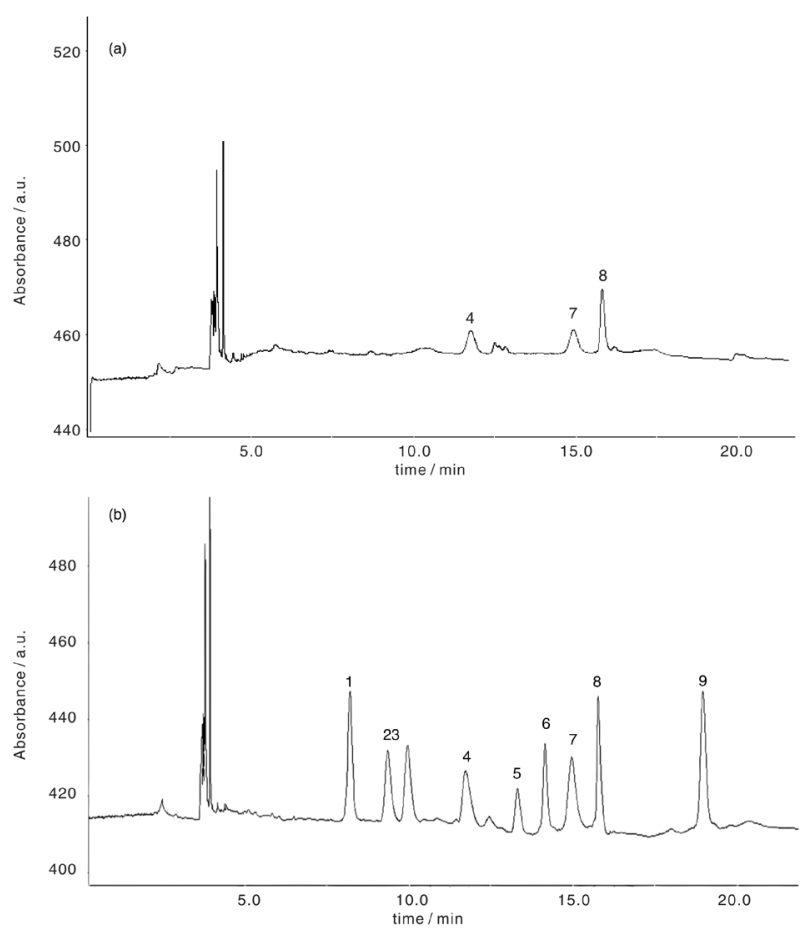

Figure 4. Chromatogram of (a) farmland soil sample solution and (b) spiked soil sample. (1) nicosulfuron; (2) thifensulfuron-methyl; (3) metsulfuro-methyl; (4) chlorsulfuron; (5) mesosulfuron-methyl; (6) tribenuron-methyl; (7) bensulfuron-methyl; (8) procarbazone sodium; (9) pyrazosulfuron-ethyl. 
The procedure of microextraction and chromatographic analysis can be completed within $30 \mathrm{~min}$. It is capable of being used in the analysis of environment samples such as surface water, conduit water and loam soil. The detection limits of the proposed method was $0.24-0.81 \mu \mathrm{g} \mathrm{L}^{-1}$ and 2.42-8.12 $\mu \mathrm{g} \mathrm{kg}^{-1}$, which can be in accordance with the other methods described in the literature, ${ }^{10,21-24}$ so do the recoveries and RSDs.

\section{Acknowledgments}

We gratefully acknowledge Prof Zeng Hongyan (Department of Sanitary Technology at the Sichuan University) for the invaluable contribution in preparing this work.

\section{References}

1. Deng, J. B.; China. J. World Pesticide 2003, 25, 24.

2. Saha, S.; Kulshrestha, G.; Int. J. Environ. Anal. Chem. 2008, $88,891$.

3. Guideline for safety application of sulfonylurea herbicides (NY 686-2003).

4. Zacharis, C. K.; Tzanavaras, P. D.; Roubos, K.; Dhima, K.; J. Chromatogr. A 2010, 1217, 5896.

5. Qi, Y.; Zhang, X.; Yang, Q.; Chu, X. G.; Chin. J. Food Hyg. 2005, 6,4 .

6. Jin, Y.; Yao, J.; Zhao, Y.; Guo, J.; Jiang, S.; Pan, W.; Liu, N.; Zhong, Y.; Insp. Quar. Sci. 2008, 6, 003.

7. Klaffenbach, P.; Holland, P. T.; J. Agric. Food Chem. 1993, 41, 396.

8. Kang, S.; Chang, N.; Zhao, Y.; Pan, C.; J. Agric. Food Chem. 2011, 59, 9776.

9. Ma, Y.; Liu, B.; Mod. Agrochem., 2011, 4, 013.

10. Xie, X.; Liao, M.; Chin. J. Pestic. Sci. 2004, 2, 11.

11. Zhang, P.; Bui, A.; Rose, G.; Allinso, G.; J. Chromatogr. A 2014, 1325, 56.
12. She, Y. X.; Cao, W. Q.; Shi X. M.; Lv, X. L.; Liu, J. J.; Wang, R. Y.; Jin, F.; Wang, J.; Xiao, H.; J. Chromatogr. B: Anal. Technol. Biomed. Life Sci. 2010, 878, 2047.

13. Asiabi, H.; Yamini, Y.; Moradi, M.; J. Supercrit. Fluids 2013 , $84,20$.

14. He, Z.; Liu, D. H.; Li, R. H.; Zhou, Z. Q.; Wang, P.; Anal. Chim. Acta 2012, 747, 29.

15. Zou, J. K.; Liu, R. Y.; Song, G.; Zhang, M. C.; Food Sci. Technol. 2009, 34, 290.

16. Ou, X. M.; Fine Chem. Intermed. 2006, 36, 1.

17. Ding, Z.; Zhang, Q.; Liu, G.; Acta Chim. Sin. 2009, 67, 1962.

18. Zanjani, M. R. K.; Yamini, Y.; Shariati, S.; Jonsson, J. A.; Anal. Chim. Acta 2007, 585, 286.

19. Leong, M. I.; Huang, S. D.; J. Chromatogr. A 2008, $1211,8$.

20. Ghonbadi, M.; Yamini, Y.; Ebrahimpour, B.; Ecotoxicol. Environ. Saf. 2015, 112,68.

21. Wang, X.; Wang, Y.; Zou, X. L.; Cao, Y.; Anal. Methods 2014, $6,2384$.

22. Zaruba, S.; Vishnikin, A. B.; Andruch, V.; Microchem. J. 2015, 122, 10.

23. Li, Y.; Peng, G.; He, Q.; Zhu, H.; Al-Hamadani, S. M. Z. F.; Spectrochim. Acta, Part A 2015, 140, 156.

24. Toraj, A. J.; Nazir, F.; Mojtaba, S.; J. Pharm. Biomed. Anal. 2014, 94,145 .

25. Leong, M. I.; Huang, S. D.; J. Chromatogr. A 2009, 1216, 7645.

26. Xu, H.; Ding, Z.; Lv, L.; Song, D.; Feng, Y. Q.; Anal. Chim. Acta 2009, 636, 28.

27. Technical Specification for soil Environmental monitoring of China (HJ166-2004).

28. Soil Testing Part 1: Soil Sampling, processing and reposition (NY/T 1121.1-2006).

29. International Union of Pure and Applied Chemistry (IUPAC); Harmonized Guidelines for Internal Quality Control in Analytical Chemistry Laboratories, 67 (4), 649, 1995.

Submitted: September 9, 2015 Published online: February 24, 2016 